\title{
Concise One-step Synthesis of 4-Arylidene-1,3-oxazolidin-2-ones with Internal Propargylic Alcohols and Isocyanates ${ }^{\dagger}$
}

\author{
Won Sun Kim, Eunyoung Yoon, Kyoung A Jo, and Eun Joo Kang \\ Department of Applied Chemistry, Kyung Hee University, Yongin 449-701, Korea. E-mail: ejkang24@khu.ac.kr \\ Received March 19, 2011, Accepted April 13, 2011
}

Key Words : 4-Arylidene-1,3-oxazolidin-2-one, Propargylic alcohol, Phenyl isocyanate, DBU

Oxazolidinones and their derivatives are widely used, as chiral synthons or chiral auxiliaries ${ }^{1}$ and as bioactive scaffolds in numerous natural products. ${ }^{2}$ Due to the importance of their structures, several methods for the synthesis of 1,3oxazolidin-2-ones have been reported. Classical methods for formation of these heterocyclic rings involve condensation reactions between corresponding amino alcohols and carbonyl precursors such as phosgene, chloroformate and dialkyl carbonates. An alternative approach is the cyclization reaction of vinyl or propargyl carbamate catalyzed by base ${ }^{3}$ or transition metal ${ }^{4}$ catalyst, in which a variety of substituted oxazolidinones are obtained under milder conditions. Tamaru, ${ }^{4 a, b}$ Gagosz, ${ }^{4 \mathrm{c}}$ and Schmalz ${ }^{4 \mathrm{~d}}$ reported the synthesis of 4-alkylidene1,3-oxazolidin-2-one from $O$-propargyl carbamates using $\mathrm{Cu}(\mathrm{I})$ and $\mathrm{Au}(\mathrm{I})$ catalyst, whereas Chandrasekaran ${ }^{3 \mathrm{~d}}$ reported $\mathrm{LiOH}$-catalyzed reaction method with DMF solvent. However, these procedures were mostly applied to the construction of 4-methylene-1,3-oxazolidin-2-one, and only a few 4-substituted methylene ones have been reported. Another method using $\mathrm{CO}_{2}$ as a carbonyl source has also been developed, ${ }^{5}$ and silver-catalyzed cycloaddition reactions involving internal propargylic alcohols and amines in supercritical $\mathrm{CO}_{2}$ and formation of 2-oxoalkylcarbamate as a reaction intermediate have been recently disclosed by Jiang. ${ }^{5 e}$ In this context, concise catalytic methods for the direct synthesis of 4-substituted methylene oxazolidinones are presented as an atom economical and environmentally benign process using internal propargylic alcohols and isocyanates.

In the first series of experiments, to overcome the lower reactivity of internal propargylic alcohols compared to terminal ones, ${ }^{5 \mathrm{e}}$ we investigated the cyclization reactions catalyzed by $N$-heterocyclic carbene catalysts, which are used for the activation of $\mathrm{CO}_{2}$ in 1,3-dioxolan-2-ones synthesis with internal propargylic alcohols. ${ }^{6}$ When the reaction was carried out with a catalytic amount $(20 \mathrm{~mol} \%)$ of triazolium catalyst $\mathbf{I}$ and DBU at $40{ }^{\circ} \mathrm{C}$, a benzylidene oxazolidinone $\mathbf{2 a}$ was obtained with $21 \%$ yield, accompanying a larger amount of carbamate intermediate 3a (Table 1). Screening tests with imidazolium catalysts II and III revealed that 1,3-diisopropylimidazolium chloride and DBU co-catalyst efficiently catalyzed the oxazolidinone cyclization reactions. Based on

This paper is dedicated to Professor Eun Lee on the occasion of his honourable retirement. these results, we performed a control experiment with only DBU base catalyst. Interestingly, we obtained highly effective synthesis of $\mathbf{2 a}$ with $94 \%$ yield. It is noteworthy that previous reports on base-catalyzed oxazolidinone synthesis featured harsh conditions such as neat pyridine at $140{ }^{\circ} \mathrm{C}$ or two-step synthesis by $O$-propargyl carbamate formation (TEA/DMAP at $90{ }^{\circ} \mathrm{C}$ ) followed by cyclization of the corresponding carbamates ( $\mathrm{LiOH}$ in DMF). The use of weaker bases under identical conditions, as described in entries 7-9, was not efficient in terms of yield of $\mathbf{2 a}$, resulting in carbamate intermediate 3a with unreacted propargylic alcohol substrate. Regarding the slightly different result with $\mathrm{KO}^{t} \mathrm{Bu}$ in entry 10 , we assume that the strong base completely and

Table 1. Optimization of conditions for benzylidene oxazolidinone synthesis ${ }^{a}$

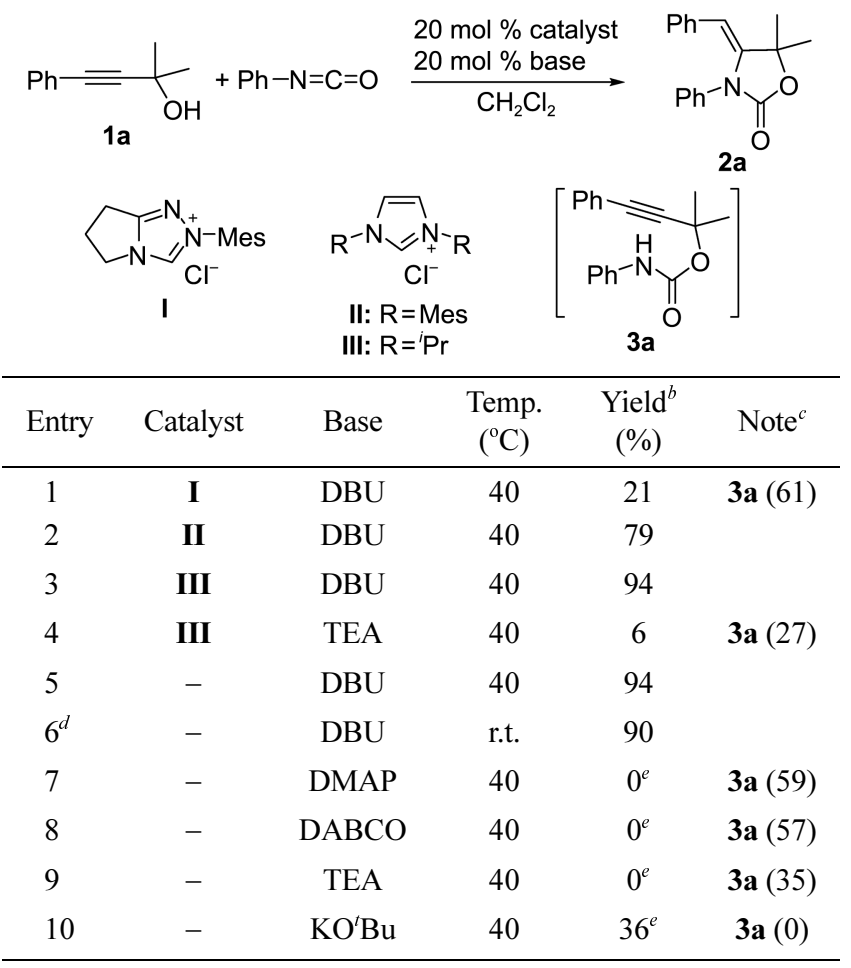

${ }^{a}$ Reactions and conditions: propargylic alcohol (1a: $\left.0.4 \mathrm{mmol}\right), \mathrm{PhNCO}$ $(0.6 \mathrm{mmol}), \mathrm{NHC}$ catalyst or base $(0.08 \mathrm{mmol}), \mathrm{CH}_{2} \mathrm{Cl}_{2}(0.2 \mathrm{M}), 12 \mathrm{~h}$, under $\mathrm{N}_{2}$, unless otherwise specified. ${ }^{b}$ Isolated yield after chromatographic purification unless otherwise specified. ${ }^{c}$ Carbamate intermediate was obtained (\% yields in parentheses). ${ }^{d}$ Reaction with $40 \mathrm{~mol} \%$ of DBU in $20 \mathrm{~h}$. ${ }^{e}$ Determined by ${ }^{1} \mathrm{H}-\mathrm{NMR}$ spectra. 
irreversibly deprotonated the alcohol protons, preventing the synthesis of carbamate 3a and efficient proton shuttling.

Having identified the ideal conditions for the preparation of 2a, we next investigated the synthesis of a variety of differently substituted oxazolidinones (Table 2). Preparation of the required internal propargylic alcohols was straightforward and was performed either by Sonogasira coupling with aryl iodide and 2-methyl-3-butyn-2-ol or alternatively via alkynylation with lithiated phenyl acetylene to various ketones. The presence of electron-donating groups conjugated to the triple bond led to an additional amount of PhNCO and DBU with an elongated reaction time (entries 2 and 3 ). The major isomer of the products was assigned by NOE measurement, and NOE interaction between the olefinic proton and methyl protons was used to identify the $(Z)$-configured isomer, indicating that addition to the alkynes proceeded predominantly in trans fashion. Internal propargylic alcohols containing electron-withdrawing halogen groups on an aryl ring were converted into corresponding oxazolidinones $\mathbf{2 d - i}$ with good to excellent yield, and the reaction of substrate 1d having a $p$-fluorophenyl group resulted in the exclusive formation of ( $Z$ )-isomer 2 d with $79 \%$ yield. Substrates bearing heterocycles such as thiophene and pyridine also were able to tolerate these reaction conditions (entries 10 and 11). We also examined the cyclization reaction involving substrates $\mathbf{1 l}$ and $\mathbf{1 m}$, which have different spatial environment due to dialkyl substituents. These substrates also afforded the expected product with excellent yield, although introduction of the isopropyl substituent led to decreased reactivity, and

Table 2. DBU catalyzed arylidene oxazolidinone synthesis: substrate scope ${ }^{a}$

$$
\mathrm{R}_{1}=\underset{\mathrm{OH}}{\stackrel{\mathrm{Me}}{\mathrm{R}_{2}}+\mathrm{PhNCO}} \underset{\mathrm{CH}_{2} \mathrm{Cl}_{2}, 40^{\circ} \mathrm{C}, 12 \mathrm{~h}}{\stackrel{20 \mathrm{~mol} \% \mathrm{DBU}}{\longrightarrow}}
$$

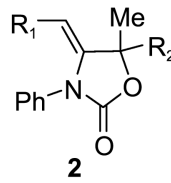

\begin{tabular}{lccccc}
\hline Entry & $\mathrm{R}_{1}$ & $\mathrm{R}_{2}$ & Product & $Z / E^{b}$ & Yield $^{c}$ \\
\hline 1 & $\mathrm{Ph}$ & $\mathrm{Me}$ & $\mathbf{2 a}$ & $86: 14$ & 94 \\
$2^{d}$ & $4-\mathrm{MeC}_{6} \mathrm{H}_{4}$ & $\mathrm{Me}$ & $\mathbf{2 b}$ & $96: 4$ & 71 \\
$3^{d e}$ & $4-\mathrm{MeOC}_{6} \mathrm{H}_{4}$ & $\mathrm{Me}$ & $\mathbf{2 c}$ & $90: 10$ & 36 \\
4 & $4-\mathrm{FC}_{6} \mathrm{H}_{4}$ & $\mathrm{Me}$ & $\mathbf{2 d}$ & $>99: 1$ & 79 \\
5 & $4-\mathrm{ClC}_{6} \mathrm{H}_{4}$ & $\mathrm{Me}$ & $\mathbf{2 e}$ & $78: 22$ & 86 \\
6 & $4-\mathrm{BrC}_{6} \mathrm{H}_{4}$ & $\mathrm{Me}$ & $\mathbf{2 f}$ & $64: 36$ & 91 \\
7 & 2- $^{d e} \mathrm{Cl}_{6} \mathrm{H}_{4}$ & $\mathrm{Me}$ & $\mathbf{2 g}$ & $69: 31$ & 79 \\
8 & 3-ClC $_{6} \mathrm{H}_{4}$ & $\mathrm{Me}$ & $\mathbf{2 h}$ & $54: 46$ & 72 \\
9 & $4-\mathrm{Cl}-2-\mathrm{F}-\mathrm{C}_{6} \mathrm{H}_{4}$ & $\mathrm{Me}$ & $\mathbf{2 i}$ & $63: 37$ & 89 \\
$10^{f}$ & $2-$ thienyl & $\mathrm{Me}$ & $\mathbf{2 j}$ & $>99: 1$ & 93 \\
11 & $2-p y r i d y l$ & $\mathrm{Me}$ & $\mathbf{2 k}$ & $62: 38$ & 58 \\
12 & $\mathrm{Ph}$ & ${ }^{n} \mathrm{Pr}$ & $\mathbf{2 l}$ & $76: 24$ & 93 \\
$13^{d e}$ & $\mathrm{Ph}$ & ${ }^{i} \mathrm{Pr}$ & $\mathbf{2 m}$ & $63: 37$ & 95 \\
14 & $\mathrm{H}$ & $\mathrm{Et}$ & $\mathbf{2 n}$ & - & 96 \\
\hline
\end{tabular}

${ }^{a}$ Reactions and conditions: propargylic alcohol $(0.4 \mathrm{mmol}), \mathrm{PhNCO}(0.6$ mmol), DBU $(0.08 \mathrm{mmol}), \mathrm{CH}_{2} \mathrm{Cl}_{2}(0.2 \mathrm{M}), 40^{\circ} \mathrm{C}, 12 \mathrm{~h}$, under $\mathrm{N}_{2}$, unless otherwise specified. ${ }^{b}$ Determined by ${ }^{1} \mathrm{H}-\mathrm{NMR}$ of isolated product mixture. ${ }^{c}$ Isolated yield of non-isolable $E / Z$ isomers after chromatographic purification. ${ }^{d}$ Reaction with 2 equiv of PhNCO and $40 \mathrm{~mol} \%$ of DBU. ${ }^{e}$ Reaction in $\mathrm{CH}_{2} \mathrm{Cl}_{2}: \mathrm{DMF}(4: 1), 24 \mathrm{~h} .{ }^{\mathrm{f}}$ Reaction in $5 \mathrm{~h}$. therefore additional amounts of PhNCO, DBU, and DMF co-solvent were needed. We also found the reaction with terminal propargylic alcohol $\mathbf{1 n}$ underwent uneventfully to afford 4-methylene-1,3-oxazolidin-2-one.

The substituents on the alkynyl and propargyl group delicately affected reactivity (Scheme 1 ). The reaction with $n$ butyl substituted alkyne 1o resulted in the corresponding carbamate formation and this result can be understood by the lower reactivity of the electron-rich alkyne moiety toward the nucleophilic addition of the primary amine. Similar result obtained with secondary propargylic alcohols suggest that dialkyl substituents on the propargyl group were indispensible to the further cyclization of corresponding carbarmate intermediates. Cleanly obtained carbamates 3o-p could be subjected to transition metal-catalyzed reactions to expand the substrate scaffold. ${ }^{4}$ Interestingly, secondary propargylic alcohol with phenyl substituent (1q) could react smoothly under the given reaction conditions, and it is noteworthy that the obtained product was 4-benzyl-5-phenyloxazol-2-one (2q'), the isomer of 4-benzylideneoxazolidin-2-one. ${ }^{5 \mathrm{~d}}$

Based on our experimental findings, a plausible mechanism for this oxazolidinone synthesis is conceived by the dual role of DBU base as follows: The carbamate intermediate is formed by an initial nucleophilic addition of the anionic oxygen of propargylic alcohol to the electrophilic carbon of isocyanate. The consecutive cyclization of carbamate intermediate is catalyzed by DBU- $\mathrm{H}^{+}$as a $\pi$-activator toward the alkyne moiety. The dual role of the conjugate acid (DBU$\mathrm{H}^{+}$) as a Brönsted acid and an alkyne-activator could give an account for the formation of the minor product, $(E)$-isomer, by the proximity effect. ${ }^{7}$

In summary, we have demonstrated that DBU base can serve as a potent catalyst for the synthesis of undiscovered arylidene oxazolidinones, and can be used for cyclization of internal propargylic alcohols and isocyanates in a mild onestep synthesis. In particular, the use of DBU has a significant advantage in oxazolidinone formation relative to previously reported basic conditions. Enantioselective hydrogenation of oxazolidinone products could build a wide vast of chiral synthons in asymmetric organic transformations. ${ }^{8}$ The knowledge gained from base-catalyzed cyclization using iso-

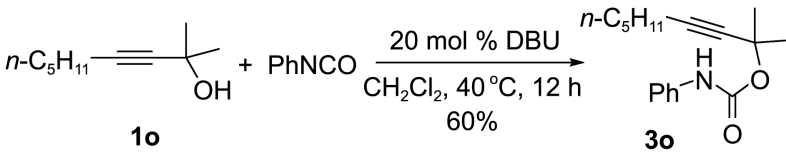

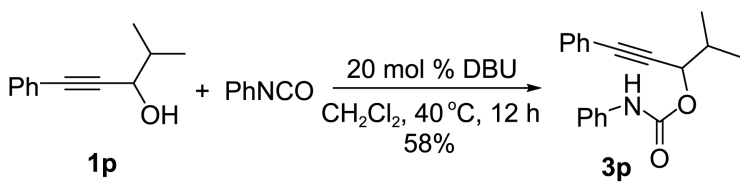

$$
\begin{aligned}
& \mathrm{Ph}=\mathrm{OH}_{\mathbf{O}}^{\mathrm{Ph}}+\operatorname{PhNCO} \underset{\mathrm{CH}_{2} \mathrm{Cl}_{2}, 40^{\circ} \mathrm{C}, 12 \mathrm{~h}}{207 \%}
\end{aligned}
$$

Scheme 1. Substituent effect on the carbamate formation and cyclization reactions 
cyanates is expected to contribute to the development of efficient nucleophilic catalysts for various heterocyclic ring formation reactions.

\section{Experimental Section}

General Procedure for Oxazolidinone Synthesis. Internal propargylic alcohol $(0.4 \mathrm{mmol})$ in distilled $\mathrm{CH}_{2} \mathrm{Cl}_{2}(0.2$ $\mathrm{M})$ was treated with $\mathrm{PhNCO}(0.6 \mathrm{mmol})$ and $\mathrm{DBU}(0.08$ mmol) at $40{ }^{\circ} \mathrm{C}$ under $\mathrm{N}_{2}$ atmosphere. After stirring for 12 hours, the reaction mixture was concentrated and purified by silica column chromatography.

2a: ${ }^{1} \mathrm{H}-\mathrm{NMR}\left(300 \mathrm{MHz}, \mathrm{CDCl}_{3}\right) \delta$ 7.06-6.98 (m, 5H), 6.90$6.82(\mathrm{~m}, 3 \mathrm{H}), 6.66(\mathrm{~d}, 2 \mathrm{H}, J=6.6 \mathrm{~Hz}), 5.63(\mathrm{~s}, 1 \mathrm{H}), 1.74(\mathrm{~s}$, $6 \mathrm{H}) ;{ }^{13} \mathrm{C}-\mathrm{NMR}\left(75 \mathrm{MHz}, \mathrm{CDCl}_{3}\right) \delta 155.3,142.3,134.9$, $133.2,129.5,128.3,128.21,128.17,127.1,127.0,126.89$, $126.86,125.8,125.7,99.8,83.1,28.2$; MS $m / z$ (EI, relative intensity) $279\left(\mathrm{M}^{+}, 100\right), 234$ (17), 220 (28), 132 (96), 117 (50); HRMS (EI) calcd for $\mathrm{C}_{18} \mathrm{H}_{17} \mathrm{NO}_{2}\left(\mathrm{M}^{+}\right)$279.1259, found 279.1257 .

2b: ${ }^{1} \mathrm{H}-\mathrm{NMR}\left(300 \mathrm{MHz}, \mathrm{CDCl}_{3}\right) \delta$ 7.06-7.01 (m, 5H), 6.66 $(\mathrm{d}, 2 \mathrm{H}, J=7.9 \mathrm{~Hz}), 6.54$ (d, $2 \mathrm{H}, J=7.9 \mathrm{~Hz}), 5.59$ (s, $1 \mathrm{H})$, $2.15(\mathrm{~s}, 3 \mathrm{H}), 1.72(\mathrm{~s}, 6 \mathrm{H}) ;{ }^{13} \mathrm{C}-\mathrm{NMR}\left(75 \mathrm{MHz}, \mathrm{CDCl}_{3}\right) \delta$ 155.3, 141.6, 135.4, 135.0, 130.2, 128.15, 128.11, 127.9, 127.7, 126.7, 125.7, 99.9, 83.1, 28.2, 21.0; MS $\mathrm{m} / \mathrm{z}$ (EI, relative intensity) $293\left(\mathrm{M}^{+}, 100\right), 278$ (3), 248 (12), 234 (41), 146 (95), 131 (48); HRMS (EI) calcd for $\mathrm{C}_{19} \mathrm{H}_{19} \mathrm{NO}_{2}\left(\mathrm{M}^{+}\right)$ 293.1416, found 293.1418.

2d: ${ }^{1} \mathrm{H}-\mathrm{NMR}\left(300 \mathrm{MHz}, \mathrm{CDCl}_{3}\right) \delta$ 7.25-7.06 (m, 3H), 7.00$6.97(\mathrm{~m}, 2 \mathrm{H}), 6.64-6.51(\mathrm{~m}, 4 \mathrm{H}), 5.57(\mathrm{~s}, 1 \mathrm{H}), 1.72(\mathrm{~s}, 6 \mathrm{H})$; ${ }^{13} \mathrm{C}-\mathrm{NMR}\left(75 \mathrm{MHz}, \mathrm{CDCl}_{3}\right) \delta 162.5,159.3,155.2,142.6$, $134.7,129.8,129.7,129.3,129.2,128.3,127.1,125.9$, $114.1,113.8,98.5,83.1,28.2 ; \mathrm{MS} m / z$ (EI, relative intensity) $297\left(\mathrm{M}^{+}, 100\right), 252$ (15), 238 (29), 165 (63), 150 (98), 135 (53); HRMS (EI) calcd for $\mathrm{C}_{18} \mathrm{H}_{16} \mathrm{FNO}_{2}\left(\mathrm{M}^{+}\right) 297.1165$, found 297.1167.

2j: ${ }^{1} \mathrm{H}-\mathrm{NMR}\left(300 \mathrm{MHz}, \mathrm{CDCl}_{3}\right) \delta$ 7.20-7.10 (m, 5H), 6.93 (d, $1 \mathrm{H}, J=5.1 \mathrm{~Hz}), 6.50-6.48(\mathrm{~m}, 1 \mathrm{H}), 5.99(\mathrm{~d}, 1 \mathrm{H}, J=3.7$ $\mathrm{Hz}), 5.60(\mathrm{~s}, 1 \mathrm{H}), 1.72(\mathrm{~s}, 6 \mathrm{H}) ;{ }^{13} \mathrm{C}-\mathrm{NMR}\left(75 \mathrm{MHz}, \mathrm{CDCl}_{3}\right)$ $\delta 155.1,142.7,135.2,129.9,128.4,127.3,126.5,125.9$, 124.6, 92.5, 83.2, 28.1; MS $\mathrm{m} / \mathrm{z}$ (EI, relative intensity) 285 $\left(\mathrm{M}^{+}, 100\right), 240$ (7), 226 (20), 138 (94), 123 (31); HRMS (EI) calcd for $\mathrm{C}_{16} \mathrm{H}_{15} \mathrm{NO}_{2} \mathrm{~S}\left(\mathrm{M}^{+}\right)$285.0823, found 285.0815.

3a: ${ }^{1} \mathrm{H}-\mathrm{NMR}\left(300 \mathrm{MHz}, \mathrm{CDCl}_{3}\right) \delta$ 7.48-7.41 (m, 4H), 7.39$7.26(\mathrm{~m}, 5 \mathrm{H}), 7.08-7.02(\mathrm{~m}, 1 \mathrm{H}), 6.58(\mathrm{br} \mathrm{s}, 1 \mathrm{H}), 1.84(\mathrm{~s}$, $6 \mathrm{H}) ;{ }^{13} \mathrm{C}-\mathrm{NMR}\left(75 \mathrm{MHz}, \mathrm{CDCl}_{3}\right) \delta 151.6,137.9,131.8$, $129.0,128.3,128.1,123.2,122.5,118.5,90.3,84.0,73.2$, 29.3; MS $\mathrm{m} / z$ (EI, relative intensity) $279\left(\mathrm{M}^{+}, 2\right), 235$ (3), 220 (4) , 160 (3), 143 (100), 128 (26); HRMS (EI) calcd for $\mathrm{C}_{18} \mathrm{H}_{17} \mathrm{NO}_{2}\left(\mathrm{M}^{+}\right) 279.1259$, found 279.1264 .

3o: ${ }^{1} \mathrm{H}-\mathrm{NMR}\left(300 \mathrm{MHz}, \mathrm{CDCl}_{3}\right) \delta 7.38(\mathrm{~d}, 2 \mathrm{H}, J=7.7 \mathrm{~Hz})$, $7.30(\mathrm{~d}, 2 \mathrm{H}, J=7.7 \mathrm{~Hz}), 7.06-7.01(\mathrm{~m}, 3 \mathrm{H}), 6.53(\mathrm{br} \mathrm{s}, 1 \mathrm{H})$, $2.21(\mathrm{t}, 2 \mathrm{H}, J=7.1 \mathrm{~Hz}), 1.72(\mathrm{~s}, 6 \mathrm{H}), 1.58-1.49(\mathrm{~m}, 3 \mathrm{H})$,
1.40-1.24 (m, 4H), $0.88(\mathrm{t}, 3 \mathrm{H}, J=6.4 \mathrm{~Hz}) ;{ }^{13} \mathrm{C}-\mathrm{NMR}(75$ $\left.\mathrm{MHz}, \mathrm{CDCl}_{3}\right) \delta 151.7,138.1,129.0,123.1,118.5,84.9$, 81.4, 73.4, 31.0, 29.6, 28.3, 22.2, 18.7, 14.0 .

3p: ${ }^{1} \mathrm{H}-\mathrm{NMR}\left(300 \mathrm{MHz}, \mathrm{CDCl}_{3}\right) \delta$ 7.48-7.40 (m, 4H), 7.347.29 (m, 5H), 7.07 (t, $1 \mathrm{H}, J=7.3 \mathrm{~Hz}$ ), 6.71 (br s, 1H), 5.52 (d, $1 \mathrm{H}, J=5.5 \mathrm{~Hz}), 2.19-2.13(\mathrm{~m}, 1 \mathrm{H}), 1.29-1.25(\mathrm{~m}, 1 \mathrm{H})$, $1.13(\mathrm{~d}, 3 \mathrm{H}, J=6.8 \mathrm{~Hz}), 1.10(\mathrm{~d}, 3 \mathrm{H}, J=7.0 \mathrm{~Hz})$.

2q': ${ }^{1} \mathrm{H}-\mathrm{NMR}\left(300 \mathrm{MHz}, \mathrm{CDCl}_{3}\right) \delta 7.58(\mathrm{~d}, 2 \mathrm{H}, J=7.1 \mathrm{~Hz})$, 7.43-7.31 (m, 7H), 7.23-7.21 (m, 2H), 7.12-7.07 (m, 2H), 7.01-6.98 (m, 2H), 3.93 (s, $2 \mathrm{H}) ;{ }^{13} \mathrm{C}-\mathrm{NMR}\left(75 \mathrm{MHz}, \mathrm{CDCl}_{3}\right)$ $\delta 154.1,136.4,135.8,133.1,130.0,129.4,129.1,128.95$, $128.87,128.8,128.4,128.2,127.9,127.8,127.7,127.0$, $124.9,121.8,104.3,29.7$.

Acknowledgments. This work was supported by the Kyung Hee University Research Fund in 2009. (KHU20090703)

\section{References}

1. (a) Gage, J. R.; Evans, D. A. Org. Synth. 1990, 68, 83; Org. Synth., Coll. Vol. VIII; Wiley: New York, 1993; 339. (b) Ager, D. J.; Prakash, I.; Schaad, D. R. Chem. Rev. 1996, 96, 835. (c) Aurelio, L.; Brownlee, R. T. C.; Hughes, A. B. Chem. Rev. 2004, 104, 5824. (d) Mukhtar, T. A.; Wright, G. D. Chem. Rev. 2005, $105,529$.

2. (a) Barbachyn, M. R.; Ford, C. W. Angew. Chem. Int. Ed. 2003, 42, 2010. (b) Reck, F.; Zhou, F.; Girardot, M.; Kern, G.; Eyermann, C. J.; Hales, N. J.; Ramsay, R. R.; Gravestock, M. B. J. Med. Chem. 2005, 48, 499. (c) Renslo, A. R.; Luehr, G. W.; Gordeev, M. F. Bioorg. Med. Chem. 2006, 14, 4227.

3. (a) Shachat, N.; Bagnell, J. J. Jr. J. Org. Chem. 1963, 28, 991. (b) Stoffel, P. J.; Speziale, A. J. J. Org. Chem. 1963, 28, 2814. (c) Francis, T.; Thorne, M. P. Can. J. Chem. 1976, 54, 24. (d) Kimura, M.; Kure, S.; Yoshida, Z.; Tanaka, S.; Fugami, K.; Tamaru, Y. Tetrahedron Lett. 1990, 31, 4887. (e) Ramesh, R.; Chandrasekaran, Y.; Megha, R.; Chandrasekaran, S. Tetrahedron 2007, 63, 9153. (f) Newton, R.; Savage, P. Aust. J. Chem. 2008, 61, 432.

4. (a) Tamaru, Y.; Kimura, M.; Tanaka, S.; Kure, S.; Yoshida, Z. Bull. Chem. Soc. Jpn. 1994, 67, 2838. (b) Buzas, A.; Gagosz, F. Synlett 2006, 2727. (c) Ritter, S.; Horino, Y.; Lex, J.; Schmalz, H.G. Synlett 2006, 3309.

5. (a) Fournier, J.; Bruneau, C.; Dixneuf, P. H. Tetrahedron Lett. 1990, 31, 1721. (b) Zhang, Q.; Shi, F.; Gu, Y.; Yang, J.; Deng, Y. Tetrahedron Lett. 2005, 46, 5907. (c) Gu, Y.; Zhang, Q.; Duan, Z.; Zhang, J.; Zhang, S.; Deng, Y. J. Org. Chem. 2005, 70, 7376. (d) Jiang, H.; Zhao, J.; Wang, A. Synthesis 2008, 763. (e) Jiang, H.-F.; Zhao, J.-W. Tetrahedron Lett. 2009, 50, 60.

6. Kayaki, Y.; Yamamoto, M.; Ikariya, T. Angew. Chem. Int. Ed. 2009, 48, 4194.

7. (a) Kundu, N. G.; Pal, M.; Nandi, B. J. Chem. Soc., Perkin Trans 1 1998, 561. (b) Kanazawa, C.; Terada, M. Tetrahedron Lett. 2007, 48, 933. (c) Park, J. H.; Bhilare, S. V.; Youn, S. W. Org. Lett. 2011, 13, 2228.

8. (a) Easton, N. R.; Cassady, D. R.; Dillard, R. D. J. Org. Chem. 1962, 27, 2927. (b) Gendre, P. L.; Jérôme, F.; Bruneau, C.; Dixneuf, P. H. Chem. Commun. 1998, 533. (c) Gendre, P. L.; Thominot, P.; Bruneau, C.; Dixneuf, P. H. J. Org. Chem. 1998, 63, 1806. (d) Shen, Z.; Lu, X.; Lei, A. Tetrahedron 2006, 62, 9237. 